\title{
88
}

\section{SIM-BEST: integrated tools for developing computer based educational simulation programs}

\author{
Maria José Marcelino \\ Teresa Mendes \\ Universidade de Coimbra \\ Portugal
}

\begin{abstract}
Educational simulations have proved their importance in the learning process. There are mainly two approaches for building these simulations: programming languages and special purpose tools. At the University of Coimbra we are developing a set of integrated tools to assist in the construction of computer based educational simulations: SIM-BEST (SIMulation: computer Based Educational Support Tools). SIM-BEST allows development of simulation programs based on different kinds of models including combined models and reusable submodels and uses essentially direct manipulation techniques, various representation, animation and interaction forms and multimedia support. It can be used individually or in a network.
\end{abstract}

Main conference themes: software

Educational areas:

Study topics:

Secondary keywords: authoring systems, courseware, modelling, simulation, tools 


\section{INTRODUCTION}

Educational computer simulations have advantages which definitely have given them a special place in learning. From primary school to college these simulations are used everywhere in the curricula though in different learning contexts and with diverse learning strategies. Educational computer simulations can be defined as educational computer programs which dynamically represent a situation described by a model with which representation the learner can interact [1].

Development of computer simulations happens under even more restrictions than the development of other kinds of educational software because simulations usually have an embedded model which must be included and sometimes even be defined. The tools used to construct these simulations therefore also must meet higher demands.

There are mainly two approaches in building educational simulations: programming languages and special purpose tools. There have been simulations developed in Pascal, C, Basic, FORTRAN, Modula-2, LISP, APL and LOGO. In the category of special purpose tools we distinguish between tools for building simulations (simulation tools) and tools for specifying and executing simulation models (modelling tools). Among the simulation tools which are by the way are not very frequently used, are SIMTEK/MOSAIKK and MacTHESIS. More common are modelling tools like DINAMIX, CMS-PC, Model Builder, STELLA, Interactive Physics and Picture Simulator. Though quite versatile and useful these only offer execution of simulation models and not construction of simulation programs. Traditional spreadsheets are in this context also used [2].

Both approaches have advantages and disadvantages. It can be generally stated that the use of programming languages leads to a more flexible product. However, this requires specialized human resources, a greater effort and longer development time. It is therefore also an expensive solution. Another disadvantage of programming languages is the difficulty in making changes. When using special purpose tools we can avoid or at least minimize all these inconveniences. These tools have the additional advantage of allowing the authors to concentrate more on the pedagogical aspects of a program than on the technical ones. We need flexible, easy to use systems which allow the implementation of programs without programming: teachers should for instance be able to build their own models or adapt existing ones. But usually, the easier to use, the more creativity is limited. It can be argued that existing systems are not versatile enough and lack some important characteristics (user friendly interface, ease of use, powerful animation techniques, rapid and intuitive 
specification, etc.). In the next section we will further explore this question on the basis of the conclusions of a comparative study.

\section{Modelling/simulation tools}

We have analyzed the modelling and simulation tools mentioned in order to find the characteristics of a general tool to assist the teacher in the construction of educational simulation programs. We chose these tools for a number of reasons among which we quote availability, price, ease of purchase, delivery time, etc. The detailed conclusions of that study can be found elsewhere [3].

We found that the metaphor used in some cases is poor (DINAMIX, CMSPC, Model Builder, SIMTEK/MOSAIKK) and in others difficult to interpret (STELLA), but also some well chosen ones (Interactive Physics, Picture Simulator).

The types of models which can be used, is sometimes limited (statical and continuous models in DINAMIX, only dynamical models in SIMTEK/MOSAIKK and MacTHESIS, only mechanical models in Interactive Physics, only some types of cellular automata in Picture Simulator). CMS-PC, Model Builder and Excel allow use of static, continuous and also some types of discrete models, while STELLA allows use of only dynamic and some types of discrete models.

Model specification is done in most of them (DINAMIX, CMS-PC, Model Builder, Excel, SIMTEK/MOSAIKK, MacTHESIS) by the direct introduction of equations which is not very good. This process should be totally graphical and interactive like in Interactive Physics and Picture Simulator. STELLA lies in between these extreme cases allowing a partial graphical specification [4].

It is not necessary to know the model resolution mechanisms for using most of them (DINAMIX, STELLA, Interactive Physics, Picture Simulator, SIMTEK/MOSAIKK, MacTHESIS). But this is not the case with CMS-PC, Model Builder and Excel. The first ones mentioned may be used in learning areas or for learning ages where the learners either do not have this knowledge or the models do not have an analytical solution (or is difficult to obtain).

In graphical representation the systems are equivalent although some perform better than others. But in terms of animation there is a lot of variation: nonexistent (DINAMIX, CMS-PC, Excel), poor (Model Builder, STELLA), reasonable (SIMTEK/MOSAIKK, MacTHESIS) and illuminating (Interactive Physics, Picture Simulator). We must however say that these two tools have a very restricted domain of application.

In general use of these system is quite easy and rapid, with sometimes different modes for the learner and for the teacher (DINAMIX, MacTHESIS). MacTHESIS and SIMTEK/MOSAIKK, the simulation tools, also allow use of 
diverse representation and interaction forms. These also have some multimedia capabilities.

From this evaluation we derived a general specification of a set of integrated tools to assist the author in the construction of educational simulations. We call these SIM-BEST (SIMulation: computer Based Educational Support Tools). These are currently under development at the University of Coimbra.

\section{SIM-BEST}

SIM-BEST main parts are three editors: the Educational Model Editor (EME), the Educational Scenario Editor (ESE) and the Educational Instruction Editor (EIE). This architecture was primary inspired by a computer simulation structure developed by Reigeluth and Schwartz [5] which is sketched in Figure 1.

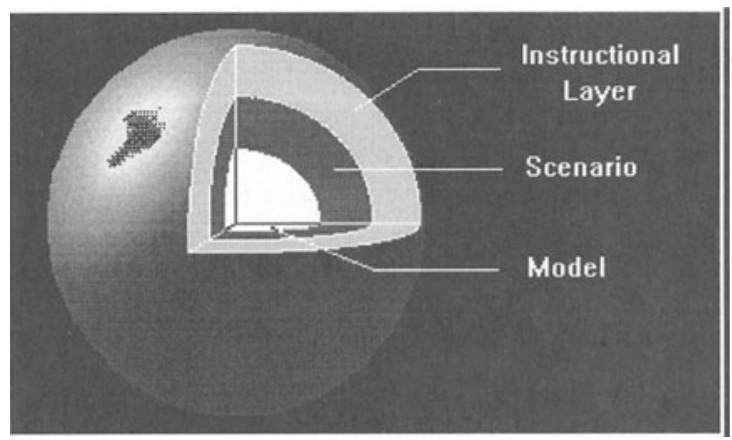

Fig. 1 General simulation program structure

A simulation is seen as a series of layers starting with a core which is the model. Around this core is another layer called the scenario, and finally we have the instructional layer. The model reflects the relationships which govern the situation being modelled. The scenario describes that situation (what happens and how, who is involved, etc.). The instructional layer tries to provide as much as possible an individualized and effective learning experience to each learner.

The Educational Model Editor (EME) allows specification and calculation of simulation models. It will be further described in the next section. The Educational Scenario Editor (ESE) allows the development of a scenario for a simulation model including use of pictures, different forms of animation (movement, change in colour, change in number, flash effects, etc.), other 
forms of representation (numeric, graphic, tabular, iconic, etc.) and change of parameters (numerical, potentiometric, spin buttons). The Educational Instruction Editor (EIE) allows the construction of an instructional layer to encapsulate the whole. The instructional layer involves the questions posed, the analysis of the answers given, feedback and help, the role of the learner and the strategy used. It is intended to allow different learning styles (exploratory, guided discovery, etc.).

At the moment the Educational Model Editor (EME) is implemented, the Educational Scenario Editor (ESE) is in an advanced stage of implementation, and what we call the Educational Instruction Editor (EIE) will be the AIDA authoring environment also developed at the University of Coimbra [6]. AIDA permits powerful and diverse forms of interaction (buttons, menus, icons, hot spots, windows, dialogue boxes, multiple choice / simple answer / open answer questions, etc.), some multimedia capabilities (sound, video) and registration of the learner's progress in order to optimize learning.

SIM-BEST uses essentially direct manipulation techniques to specify the models, the scenarios and the instructional layers aiming at easy and intuitive use. Another important feature of SIM-BEST is that it allows the combination of the same model with different scenarios and/or different learning layers. In this way use of both tools and the products which will originate from them, will be optimized. It also allows construction of submodels (program chunks) which can be reused later (this eases the construction task and promotes sharing among authors).

SIM-BEST is developed for Windows which allows portability, but also language and cultural portability problems will be addressed just as in the AIDA authoring environment. SIM-BEST will support network use permitting team development of applications.

\section{The Educational Model Editor}

\section{Various explicit models}

The Educational Model Editor (EME) supports specification and execution of static, continuous, discontinuous-change and discrete models, and also of combined type models. Static models are just given by algebraic equations. Continuous models are characterized by continuous variation of some variables and their derivatives in relation to other(s). These models are typically described by differential equations although algebraic equations may be included. In EME first order continuous models and higher order models may be specified. Discontinuous-change models are continuous models with discontinuities (sudden changes in the state of the model). Discrete models are models described by changes which occur in 'jumps' and not continuously. 
We are talking essentially about models which can be effectively separated from the program these are embedded in and which may be formalized as a set of equations, or a series of events; we call these explicit models. Existing authoring systems like HyperCard and Authorware do not allow use of such explicit models because these do not make calculation of the model possible through for example numerical integration methods or event processing mechanisms.

\section{Description of EME}

EME can both operate as a stand-alone tool and in conjunction with the scenario editor (ESE) and the instruction editor (EIE). In Figure 1 each layer shown can actually produce different output:

- a model which the learner can build and change;

- a quasi demonstration type of simulation with which the learner can interact freely at parameter level, but without any learning strategy behind;

- a simulation through which the learner is 'guided' in order to make the learning experience more effective.

In using these editors separately or in an integrated way different learning modes and strategies can be realized. There are authors defending one mode of learning against others, but we think that all should have a place in learning. The choice depends on the objectives to be achieved, the topic covered, the target audience, etc. There are so many variables in each learning experience that we prefer to be able to choose the adequate option in each situation.

A model is described in an iconic diagram which specifies the model entities and their links, using direct manipulation techniques. Continuous entities mainly are quantities and rates. Discrete entities can be servers, clients, queues, conveyors, semaphores and activities. A model entity can also be defined in tabular or graphical form permitting integration of data from other tools, like a spreadsheet, or from experimentation [7].

Concerns continuous models EME provides several integration methods for differential equations. The Euler method and two Runge-Kutta methods seem to suffice for a tool of this kind. In discontinuous-change models the discontinuities can be of two types: time-event (the trigger of the discontinuity is time dependent) or state-event (triggered by the state of a model variable). In terms of discrete models an event-driven technique is used to solve the models. For the calculus of combined models a hybrid technique is used.

EME allows several forms of representation of model behaviour, such as tabular and graphical. A trial can be made to choose which entities to represent in each form, the number format, the graph type (linear, points, bars, etc.), the scale adjustment (automatic or manual), etc. 
The independent variable can be time which is the case for dynamic models, a very important class of continuous models, or another as specified by the user (for example distance for the study of steady-state field problems). This feature is not included in STELLA, SIMTEK/MOSAIKK and MacTHESIS. However DINAMIX, Model Builder and CMS-PC offer it.

To illustrate the use of EME, let us consider three very simple simulation models. The first one is a pure continuous model, the second is a discontinuous-change model which uses the first model as a sub-model, and the third is a discrete model with an adaptation of the first model included in its definition.

$$
\begin{array}{ll}
\frac{\mathrm{dRoomT}}{\mathrm{dt}}=- \text { Heat } & \text { (room temperature) } \\
\text { Heat }=\frac{\text { RoomT }- \text { OutT }}{\text { HT_C }} & \text { (heat transfer) } \\
\text { OutT }=5 & \text { (outdoor temperature) } \\
\text { HT_C }=6 & \text { (heat transfer constant) }
\end{array}
$$

Fig. 2 A simple continuous model

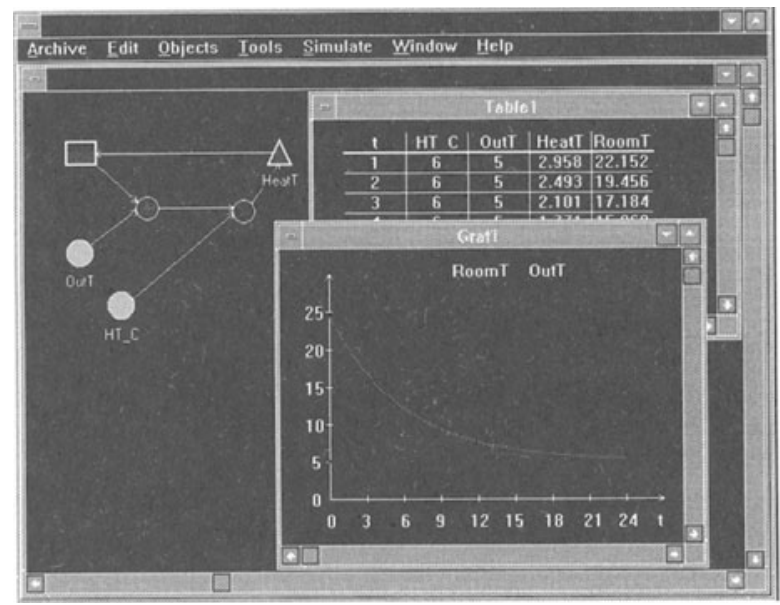

Fig. 3 Educational Model Editor (EME) with the room temperature example 
First we consider the evolution of the temperature in a room where the heating is suddenly turned off [8]. Let us assume that the outdoor temperature is $5^{\circ} \mathrm{C}$ and that the room temperature is $25^{\circ} \mathrm{C}$. Later these can be changed and the model run again. This physical situation can for instance be described by the equations in Figure 2.

In Figure 3 we see these equations specified in EME along with a tabular and a graphical representation of the model behaviour.

Now suppose that we want to start modelling our system, the room, some time before the heating is turned off. The room temperature is stabilized on $25^{\circ} \mathrm{C}$ and the outdoor temperature is still $5^{\circ} \mathrm{C}$. This is a discontinuous-change model and we want to see the transfer from one situation to the other. Actually there are two models, or sub-models, describing this system. Figure 4 shows SubMod 2 which is exactly the model described above, while SubMod1 can be described by just two algebraic equations (RoomT $=25$ and OutT $=5$ ). The transfer from the one to the other can be triggered by a user action (pressing the button of the heater, for instance). This example cannot be modelled in either of the other tools mentioned in this paper.

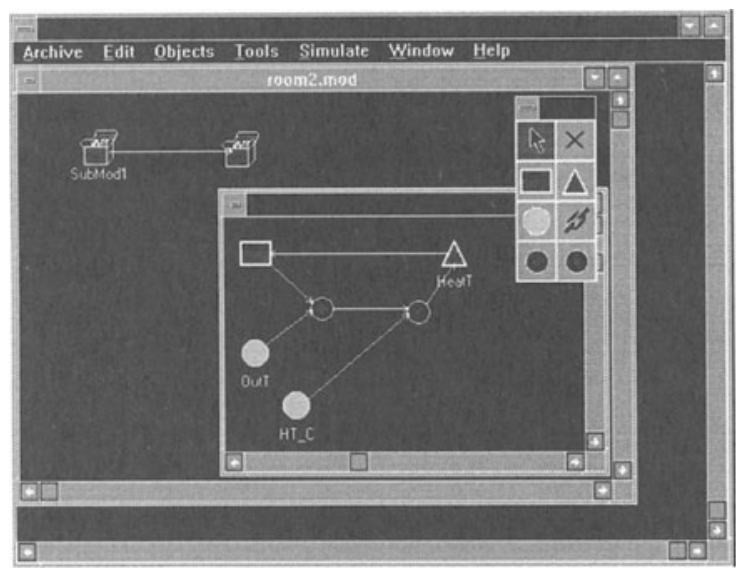

Fig. 4 Educational Model Editor (EME) with the room temperature example using submodels

The last example is a discrete model representing steel ingot heating in a soaking pit furnace in a steel plant (Fig. 5) [9]. Let us assume that the furnace has a capacity of 9 ingots and when full, the arriving ingots have to wait in a queue until there is room in the furnace. Ingot temperature inside the furnace can be 
described by a model very similar to the first one we used but with some adaptations. EME allows us to reuse models previously developed by others; something we regard as a very strong point. As can be seen from the figure IngotT is very similar to RoomTmp; some entity names, RoomT and OutT, have been changed to IngT and FurnaceT, and the sign of the derivative has been changed to a minus sign (the model here describes a heating process instead of a cooling one).

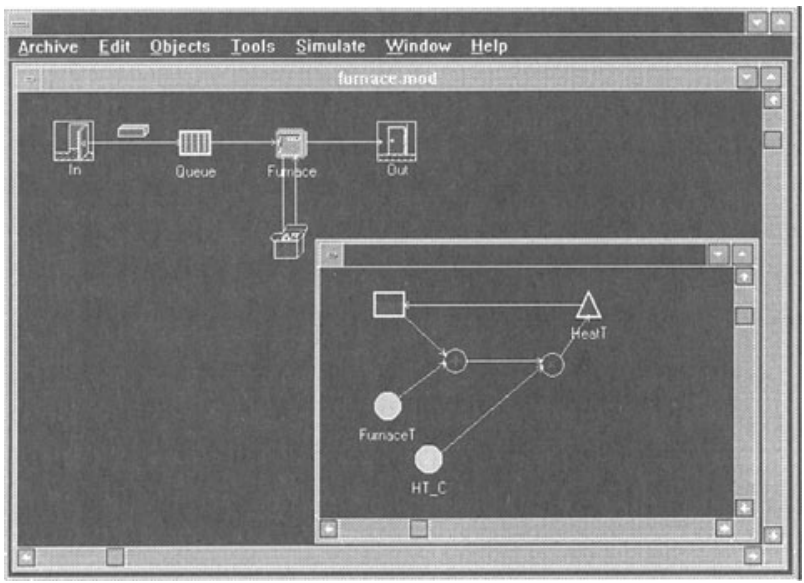

Fig. 5 Educational Model Editor (EME) showing the steel plant example

\section{PRELIMINARY EVALUATION}

Some preliminary evaluation of the ease of use of SIM-BEST was done by teachers. The evaluation essentially concerned time and effort to learn to use the tools, time and effort to develop a model, and time and effort to reuse/adapt models. The evaluation results were quite encouraging, but a lot is still to be done. Field evaluation with students is planned to take place shortly.

\section{CONCLUSION}

In this paper some features of SIM-BEST (SIMulation: computer-Based Educational Support Tools) have been described. We believe that teachers need this kind of support tools to make efficient and effective construction, maintenance and utilization of educational software programs possible, as the 
market will not provide these programs. It is generally accepted that the development and maintenance of software, especially educational software, are very time-consuming and expensive activities and this seems particularly true for educational simulations. We believe that our own and other tools can support and stimulate our teachers in such demanding tasks.

\section{SOFTWARE MENTIONED}

SIMTEK/MOSAIKK is a product of the Royal Norwegian Ministry of Education and Research.

MacTHESIS is a product of the University of Twente, Faculty of Educational Science and Technology, Instrumentation Technology, the Netherlands.

DINAMIX is a product of GEP, Portugal.

CMS-PC is a trade mark of Longman Micro Software.

Model Builder is a product of the Advisory Unit for Microtechnology in Education.

STELLA is a trade mark of High Performance Systems, Inc. Interactive Physics is a trade mark of Knowledge Revolution. Picture Simulator is a product of Newsoft, UNINOVA, Portugal. Excel is a product of Microsoft Corp..

\section{REFERENCES}

1. Mendes, T. (1988) Impact of New Information Technologies on Technical Possibilities for Educational Computing in School Environments, presented at the EURIT Conference.

2. Przasnyski, Z.H. (1989) On Using Spreadsheets to Model Decision Problems. Computers \& Education, 13 (2) pp. 117-128.

3. Marcelino, M. J. and Mendes, T. (1994) Estratégias e Ferramentas para a Construção de Programas Educativos de Simulação, in II Congresso Iberoamericano de Informática na Educação Proceedings, (eds DEPGEF Ministério da Educação), Vol 2, pp. 41-48.

4. Miller, R., et al. (1993) Educational Tools for Computational Modelling. Computers \& Education, 21 (3) pp. 205-261. 
5. Reigeluth, C. M. and Schwartz, E. (1989) An Instructional Theory for the Design of Computer-Based Simulations. Journal of Computer-Based Instruction, 16 (1) pp. 1-10.

6. Mendes, A. J., Orvalho, J. and Mendes, T. (1994) Developing Educational Software with the AIDA Authoring Environment, in 19th International Conference with Summer School Information Technologies and Programming Proceedings, (ed. Barnev, P.) pp. 139-146.

7. Haugen, H. (1990) Systems Dynamics as an Educational Method, in The Fifth World Conference on Computers and Education Proceedings, (eds. McDougall, A. and Dowling, C.), Elsevier Science Publishers B. V., NorthHolland, pp. 115-120.

8. Byrknes, A.H. and Myrtveit, M. (1990) Mosaikk and Simulation, UFDDatasekretariatet, the Royal Norwegian Ministry of Education and Research, (eds. ModellData A/S), Manger, Norway.

9. Fayek, A.M. (1988) Introduction to Combined Discrete-Continuous Simulation Using SIMSCRIPT II.5, (eds. CACI Products Company), La Jolla, California. 